\title{
Multiplane Encoded Light-Sheet Microscopy for Enhanced 3D Imaging
}

\author{
Alessandro Zunino, Francesco Garzella, Alberta Trianni, Peter Saggau, Paolo Bianchini, \\ Alberto Diaspro,* and Martí Duocastella*
}

Cite This: ACS Photonics 2021, 8, 3385-3393

Read Online

\section{ACCESS | Lill Metrics \& More | 回 Article Recommendations ｜（） Supporting Information}

ABSTRACT: Light-sheet microscopes have become the tool of choice for volumetric imaging of large samples. Based on a wide-field acquisition scheme, they are capable of optical sectioning at diffraction-limited resolution and minimal overall photodamage. Unfortunately, traditional architectures are limited in speed because 3D images are collected by either sample translation or synchronized movement of both light-sheet and detection objective lens. A promising solution avoiding slow mechanical movements is to extend the depth-of-field of the microscope and moving only the light-sheet. However, this normally comes at the cost of losing light and contrast, compromising the signal-to-noise ratio of the images. Here, we propose an innovative technique

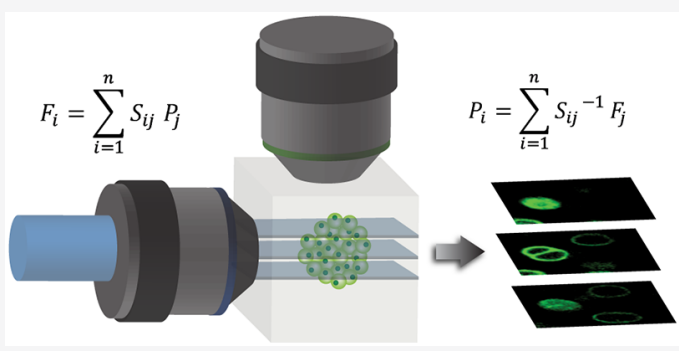
devoted to restoring the quality of the images, while preserving the speed of extended depth-of-field microscopes. It is based on generating a stack of parallel light-sheets using a pair of orthogonal acousto-optic deflectors, enabling the simultaneous illumination of different sample planes. Given the extended depth-of-field, all such planes appear in focus and can be acquired in a superimposed single frame. By applying a single-step inversion algorithm, we can decode a stack of frames into a volumetric image whose signal-tonoise ratio and contrast are greatly enhanced. We provide a detailed theoretical framework of the method and demonstrate its feasibility with volumetric images of kidney cell spheroids.

KEYWORDS: optical microscopy, volumetric imaging, extended depth-of-field, acousto-optics, phototoxicity, signal-to-noise ratio

$\mathrm{O}$ ne of the main goals of optical microscopy is retrieving quantitative and volumetric information while minimizing sample photodamage. This requires three-dimensional (3D) imaging techniques with high spatiotemporal resolution and optical sectioning capabilities. Among the existing techniques, light-sheet fluorescence microscopy (LSFM) has become the tool of choice for volumetric imaging of large samples, ${ }^{1}$ offering a unique combination of speed, field-of-view (FoV), and spatial resolution. ${ }^{2}$ In LSFM, the sample is illuminated with a light-sheet ${ }^{3}-a$ thin (along the $z$-axis) and wide (in the $x-y$ plane) beam of light-produced by a cylindrical lens or a rapidly scanning Gaussian, Bessel, or Airy beam. $^{4-6}$ An objective lens, mounted orthogonally to the illumination plane, collects the fluorescent light, and the corresponding image is recorded with a camera. ${ }^{7}$ The combination of a wide-field acquisition scheme with shaped illumination allows fast optical sectioning over a large FoV, at diffraction-limited resolution while using low light doses. Unfortunately, traditional architectures are not optimized for fast 3D imaging. Typically, volumetric imaging in LSFM is performed by acquiring a sequence of $2 \mathrm{D}$ images from different focal planes of the sample, the so-called $z$-stack. Since the lightsheet and the focal plane of the detection lens must coincide to avoid blurring or image artifacts, collection of a $z$-stack is obtained either by sample translation or by synchronized movement of light-sheet and objective lens. In both cases, a slow mechanical movement is needed, constraining the maximum acquisition rate. In order to overcome this limitation, various innovative LSFM architectures have been proposed. For instance, remote focusing detection enables axial scanning at high rates without introducing significant aberrations. ${ }^{8,9}$ While offering substantial speed advantages, its practical usage is hindered by the complexity of setup and alignment procedures. The combination of a light field camera-which exploits an array of microlenses to distinguish the axial origin of the light-with a thick light-sheet can be used to retrieve information on a whole volume from a single frame. ${ }^{10,11}$ However, the reconstruction algorithm needed for this solution is computationally demanding, and the resulting images typically have degraded lateral resolution. Another possibility is to acquire fewer frames per stack and reconstruct the volume using a compressed sensing algorithm, thus reducing the total acquisition time. ${ }^{12}$ Still, this strategy offers

Received: September 13, 2021

Published: November 3, 2021 

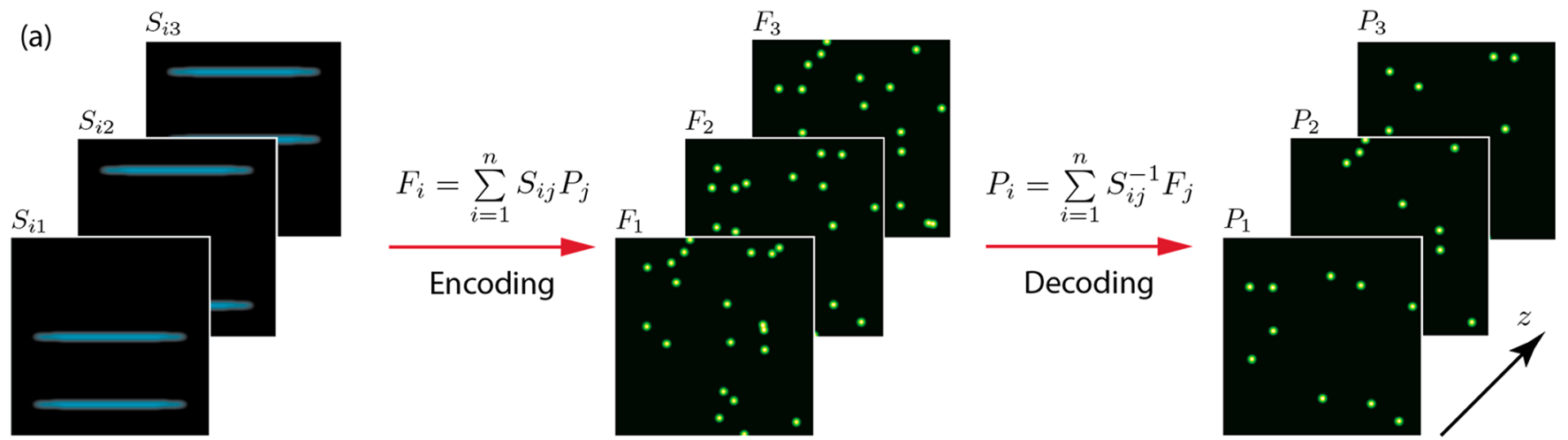

(b)

Hadamard
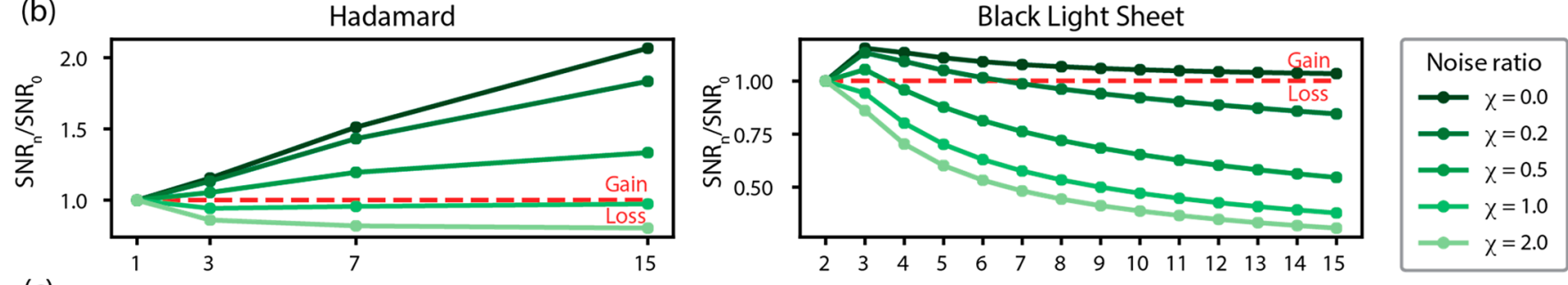

(c)

$\mathrm{n}$
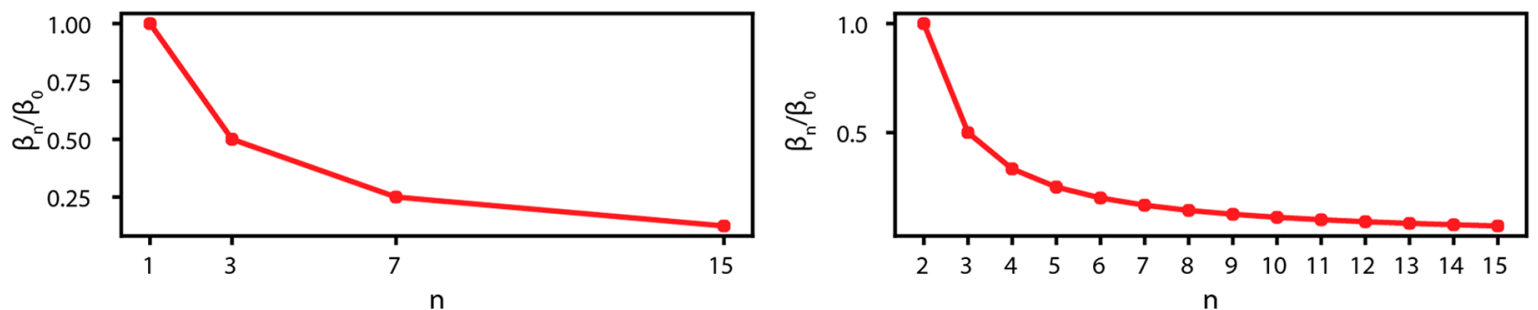

Figure 1. (a) Working principle of multiplane encoded light-sheet microscopy. A set $S_{i j}$ of parallel light-sheets is shined on the sample, illuminating at the same time multiple planes $P_{j}$ whose superposed images are collected as the frame $F_{i}$. In order to retrieve the individual images, one needs to calculate the inverse of the encoding sequence $S_{i j}^{-1}$. (b) SNR of the decoded images acquired with the Hadamard encoding (left) and black lightsheet encoding (right), seen as functions of the number of acquired frames. Both plots are normalized by the SNR of a sequential scan. (c) Background of the decoded images acquired with the Hadamard encoding (left) and black light-sheet encoding (right), seen as functions of the number of acquired frames. Both plots are normalized by the background level of a sequential scan.

only a limited speed improvement, and it is likely to generate artifacts when the sample is not sparse. Alternatively, it is possible to extend the depth-of-field (DoF) of the detection objective. With this approach, all the sample planes appear in focus, and the images can be acquired by only moving the light-sheet, with no need for mechanical synchronization. Notably, translation of the light-sheet can be performed in a few milliseconds, greatly increasing imaging speed. Several techniques exist for generating an extended depth-of-field (EDoF), including the use of spherical aberrations, ${ }^{13}$ wavefront coding, ${ }^{14,15}$ and varifocal lenses. ${ }^{16-18}$ Unfortunately, EDoF techniques can produce a significant loss of photons per plane (see Figure S1). As a result, the acquired $z$-stack exhibits a degraded signal-to-noise ratio (SNR).

A straightforward solution is to increase the excitation intensity. While the high power of current lasers enables this implementation in almost any EDoF light-sheet system, this approach faces two major drawbacks. First, the fluorophore response is nonlinear and, above their saturation threshold, no additional fluorescence signal is gained from increasing illumination levels ${ }^{19}$ (see Figure S2). Second, and more importantly, the higher the peak intensity, the greater the risk of photodamaging the sample-an enormous detriment for live microscopy. To prevent phototoxicity and gain SNR, increasing the exposure time of the images while keeping the illumination levels low is preferrable..$^{20,21}$ However, this irremediably comes at the cost of sacrificing acquisition speed. An alternative solution is parallelizing the illumination to increase the information captured per frame. In this case, several planes are simultaneously illuminated; thus, the exposure time of individual planes can be increased without loss of acquisition speed. Although a common practice in laser scanning microscopy, such a multiplexing strategy remains difficult to implement in LSFM. Recent attempts include multibeam interference ${ }^{22}$ and arrays of incoherent and frequency-modulated light-sheets, ${ }^{23}$ but they can be limited by the speed of spatial light modulators or require instrumentspecific components not commercially available. So far, a lightsheet parallelization method that obviates mechanical moving parts and rapidly produces enhanced SNR volumetric images has not been reported.

Here, we propose a novel and straightforward technique that fills this void and produces images with enhanced signal-tonoise/background-ratio in EDoF Light-Sheet microscopes. It is named multiplane encoded light-sheet microscopy (MELM) and is based on illuminating a sample with encoded sequences of simultaneous multiple light-sheets. ${ }^{24,25}$ As shown in Figure $1 \mathrm{a}$, each sequence contains information from multiple planes. The sum of the corresponding images is collected in a single camera frame. By acquiring different frames-as many as 
sample sections desired to capture-images from individual planes can be decoded using a computationally inexpensive single-step reconstruction algorithm. Notably, the decoded images exhibit enhanced SNR depending on the illumination sequence used. Additionally, volumetric acquisition can occur at the same rate as in standard EDoF-LSFM, thus preserving the core advantages of this technique in terms of speed and ease of implementation. In this manuscript, we provide a full theoretical framework to describe our method. Supported by numerical simulations, we demonstrate the feasibility of our instrument, and we quantify the gain in SNR with imaging experiments using fluorescent beads and biological samples.

\section{SIGNAL-TO-NOISE RATIO INCREASE THROUGH PARALLELIZATION}

The fundamental hypothesis of MELM is that parallelized illumination using tailored light sequences can enhance the SNR in EDoF-LSFM. Mathematically, we can describe such a process as follows. Let $\mathbf{P}$ be the $1 \times n$ array of images composing the full $z$-stack, and $\mathbf{F}$ be the $1 \times n$ array of acquired camera frames. Then, we can write the acquisition process as

$$
F_{i}=\sum_{j=1}^{n} S_{i j} P_{j}+e_{i}
$$

where $S_{i j}$ is a coefficient whose value is 1 when the plane $P_{j}$ is illuminated by a light-sheet and 0 when not, and $e_{i}$ is the noise. ${ }^{26}$ The above equation can also be represented using the following matrix form:

$$
\mathbf{F}=\mathbf{S P}+\mathbf{e}
$$

Here, $\mathbf{S}$ is an $n \times n$ matrix whose rows describe the encoding sequences of on/off light-sheets for each frame. If $S$ is invertible and well-conditioned, we can find an estimate of the images $\mathbf{P}$ by directly inverting the previous equation:

$$
\hat{\mathbf{P}}=\mathbf{S}^{-1} \mathbf{F}=\mathbf{P}+\mathbf{S}^{-1} \mathbf{e}
$$

Notably, MELM enables decoding $n$ planes $P_{j}$ from $n$ frames $F_{j}$, whose noise content is now $\mathbf{S}^{-1} \mathbf{e}$ instead of e. To make this transformation advantageous, the signal-independent noise must be shared among multiple illuminated planes. Consequently, the larger the number of simultaneously acquired planes, the lesser the noise per plane. However, the decoding step needed to retrieve information from each plane can return some noise to the images. The amount of added noise depends on the encoding matrix. Therefore, the choice of $\boldsymbol{S}$ is crucial in determining the SNR of the decoded images. To illustrate this effect, we used three different illumination patterns, each one represented by a unique matrix:

1. Sequential Scan. This corresponds to the traditional implementation of LSFM, in which a single light-sheet illuminates a single plane, and it is moved to a different axial position at each frame. In this case, the encoding matrix is the identity $\mathbf{I}$. Consequently, $\mathbf{S}^{-1}=\mathbf{I}=\mathbf{S}$ and the noise content is unchanged. This nonparallelized acquisition scheme is used as a benchmark for the following multiplane schemes.

2. Black Light-Sheet. As its name indicates, it is based on using $n-1$ parallel and identical light-sheets to simultaneously illuminate a volume divided into $n$ sections. In other words, all planes within a volume are illuminated except for one. The axial position of the dark plane is changed after each frame. The corresponding matrix $\mathbf{S}$ contains 0 s on the main diagonal and $1 \mathrm{~s}$ everywhere else. The expected gain in SNR for an image in the $z$-stack is

$$
\frac{\mathrm{SNR}_{\mathrm{n}}}{\mathrm{SNR}_{0}}=\sqrt{\frac{1+\chi^{2}}{1+(n-1) \chi^{2}}} \frac{(n-1)}{\sqrt{\left(n^{2}-3 n+3\right)}}
$$

where $\chi$ is the ratio of the standard deviation of the photon noise to the standard deviation of the camera noise of each plane, ${ }^{27}$ and $\mathrm{SNR}_{0}$ is the SNR of the image acquired using a sequential scheme with the same exposure time and peak intensity per light-sheet (see Supporting Information for a full derivation). The parameter $\chi$ depends on several variables, such as detector type, brightness of the sample, and exposure time. In fast imaging experiments, the last variable is expected to be low, resulting in a small number of collected photons and, correspondingly, a low value of $\chi$.

3. Hadamard Scan. A volume, divided into $n$ planes, is simultaneously illuminated by $(n+1) / 2$ identical light-sheets. At each frame, the axial positions of the light-sheets are changed according to the rows of the Hadamard matrix $S .^{28}$ In this case, the expected SNR gain is

$$
\frac{\mathrm{SNR}_{n}}{\mathrm{SNR}_{0}}=\sqrt{\frac{1+\chi^{2}}{1+\frac{n+1}{2} \chi^{2}}} \frac{n+1}{2 \sqrt{n}}
$$

which also depends on the parameter $\chi$. Note that Hadamard matrices are defined only for $n=2^{k}-1$, with $k>1$ and integer, differently than black light-sheet matrices that can be defined for every natural number $n$ (see Supporting Information).

Figure $1 \mathrm{~b}$ shows plots of the SNR enhancement for the black light-sheet and Hadamard encoding strategies as a function of the number of illuminated planes and photon noise. As expected, when the photon noise is negligible, both cases provide a gain in SNR over the traditional sequential scan. Such a gain increases with the number of planes for the Hadamard case-it scales as $\sqrt{n} / 2$. Instead, when using the black light-sheet encoding, the SNR is maximized when 3 planes are illuminated and then asymptotically approaches the same value as the sequential scan. As the photon noise content increases, both cases exhibit a reduction of the SNR advantage. However, the two methods have a different tolerance to photon noise, with the Hadamard scan preserving its benefits for higher values of $\chi$ than the black light-sheet. These observations are valid assuming the detector is capable of collecting the additional light coming from all the illuminated planes. In other words, the dynamic range of the camera must be sufficient to gather all this information. Given that parallelization is useful to improve the SNR of images when the signal is low, this assumption is valid in the vast majority of practical situations in fluorescence bioimaging.

Microscopy images are not only affected by noise, but they typically suffer from the presence of background. This latter has originated from various contributions such as out-of-focus fluorescence (signal-dependent background) or detector dark current and environmental light (signal-independent background). EDoF-LSFM inherently rejects the first type of background but can still suffer from the signal-independent contributions that we define as $\beta$. The parallelization of MELM also enables reduction of this value, thus increasing the contrast of the reconstructed images. In particular, the expected background reduction with the black light-sheet encoding is 
(a)

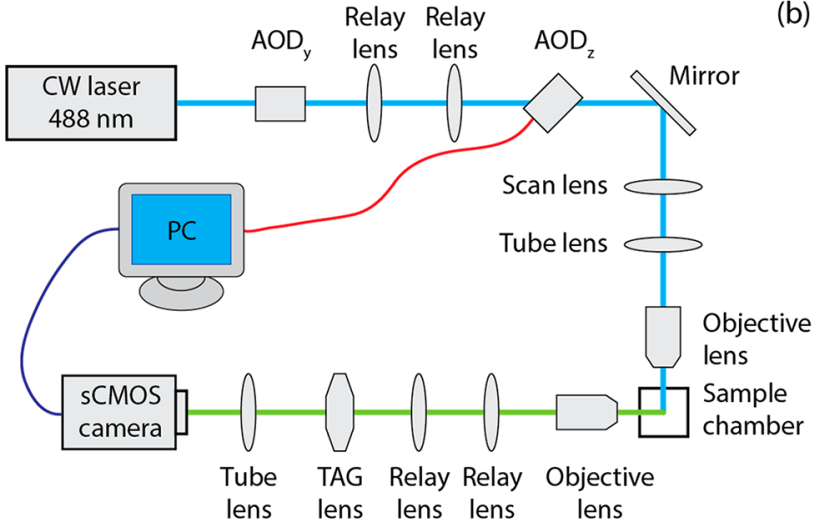

(b)

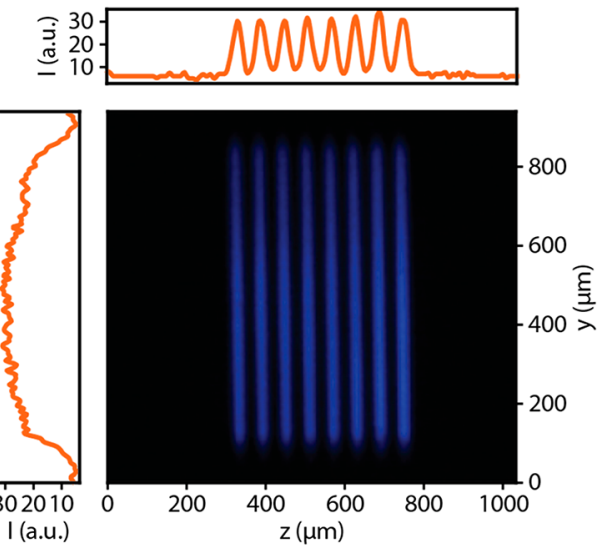

(d)
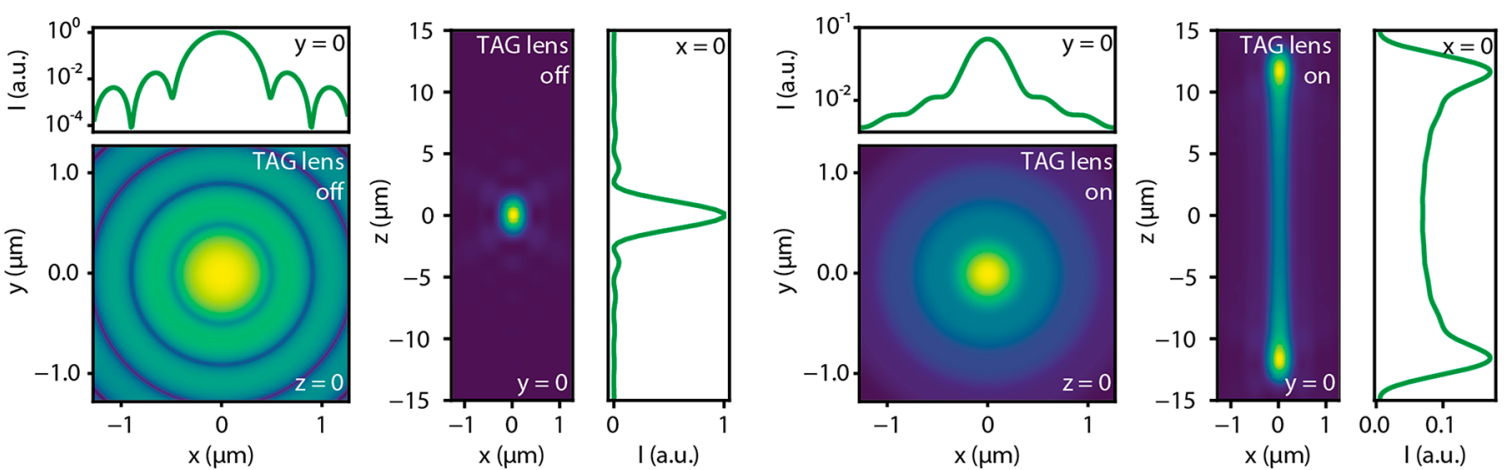

Figure 2. (a) Sketch of the experimental setup. The blue line defines the excitation arm, and the green line defines the detection arm. (b) Experimental image of light-sheets taken at the focal plane of the scan lens. On top, the intensity profile along the $z$-axis shows that the light-sheets have comparable intensity. On the left, the intensity profile along the $y$-axis shows a good longitudinal homogeneity. (c) Native PSF of the detection objective lens $(40 \times / 0.8 \mathrm{NA})$ calculated with the Born and Wolf model. The image of the $x y$ plane at $z=0$ and the corresponding intensity profile are in a logarithmic scale. The DoF, defined as the fwhm along the $z$-axis, is $1.5 \mu \mathrm{m}$. (d) Extended PSF, obtained by scanning the native PSF along the $z$-axis with the TAG lens. In this example, the DoF extension is about $25 \mu \mathrm{m}$, but it can be tuned by applying a different voltage drive to the TAG lens. The intensity scale is the same as in (c). It can be seen that the lateral width is preserved, but the intensity per plane is reduced.

$$
\frac{\beta_{n}}{\beta_{0}}=\frac{1}{n-1}
$$

where $\beta_{0}$ is the background of the image acquired using the sequential scan. In the case of the Hadamard scan, such a reduction can be written as

$$
\frac{\beta_{n}}{\beta_{0}}=\frac{2}{n+1}
$$

As shown in Figure 1c, both encoding methods enable reducing the background with respect to the sequential scan. In addition, such a reduction increases with the number of illuminated planes. However, the black light-sheet approach has a better performance in background suppression. Therefore, while the Hadamard scan provides the best SNR gain of the strategies analyzed, the black light-sheet can be preferable when a strong background is present. Note that it is always possible to choose and tune the optimal encoding method that best suits the target application or sample.

\section{MELM IMPLEMENTATION}

MELM can be implemented in any traditional or inverted light-sheet microscope, with two key modifications: first, incorporating a method for generating an array of parallel and identical light-sheets that can be individually switched on and off; second, applying a technique to extend the DoF of the detection objective lens. In our microscope, presented in Figure 2a, we use a pair of acousto-optic deflectors (AODs), optically conjugated in a $4 \mathrm{f}$ system, to generate multiple lightsheets. The first AOD-driven with a sinusoid, the frequency of which is modulated by a triangular function-periodically deflects a Gaussian beam along the $y$-axis, thus generating a light-sheet when watched at the frame speed of the camera detector. ${ }^{29}$ This approach, compared to the more traditional cylindrical lens, allows the illumination to be confined to a region of interest, thus optimizing the photon budget. ${ }^{30} \mathrm{We}$ use the second AOD to generate the encoded illumination sequences. To this end, we drive it with multiple sine waves $V_{i}$ while collecting the superpositioned frames $F_{i}$ :

$$
V_{i}(t) \propto \sum_{j=1}^{n} S_{i j} \sin \left(\omega_{j} t\right)
$$

The amplitudes $S_{i j}$ are exactly the entries of the encoding matrix $\mathbf{S}$, and each acoustic frequency $\omega_{j}$ diffracts the light to a different axial position, ${ }^{31}$ thus obtaining a stack of parallel lightsheets illuminating the planes $P_{j}$ only if $S_{i j}=1$. As shown in Figure $2 \mathrm{a}$ and in the SI video, the illumination pattern produced with this technique shows good uniformity along the $y$-axis, and the intensity of each beam is the same. Notably, the good uniformity of the frequency response of the AOD and the 
(a)
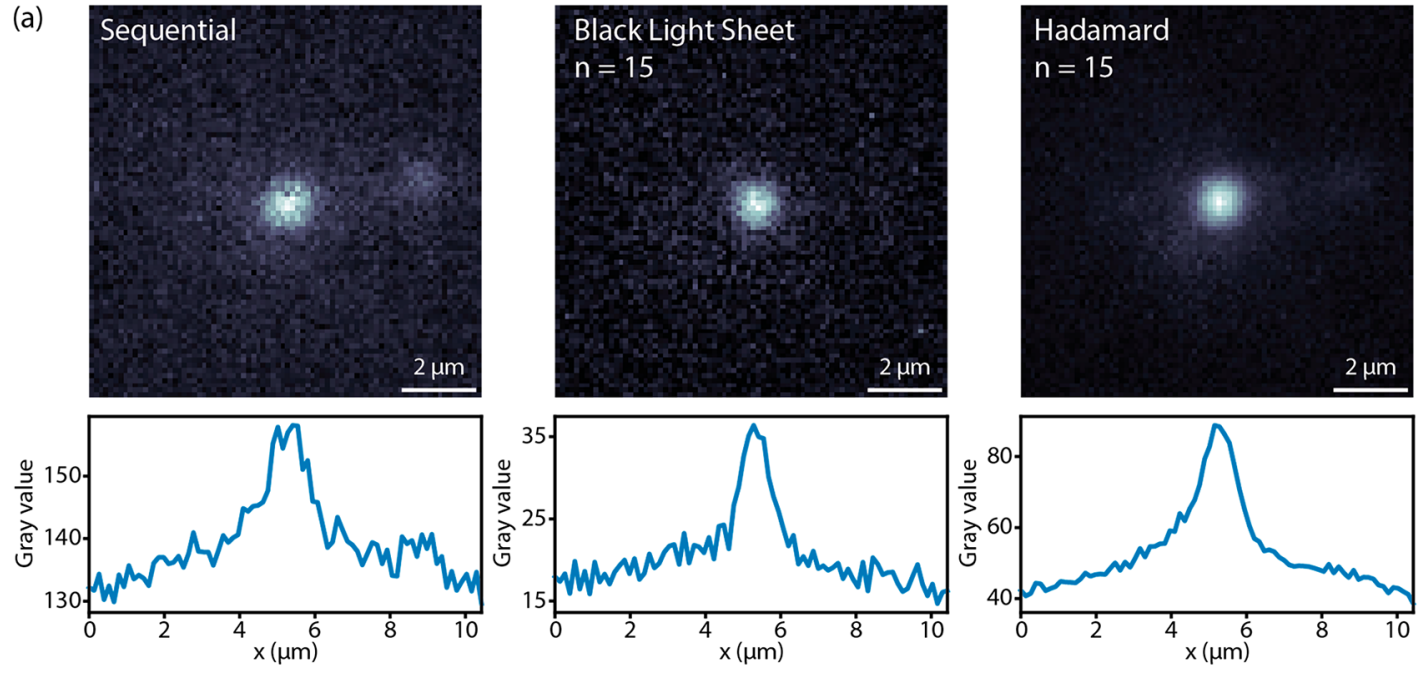

(b)

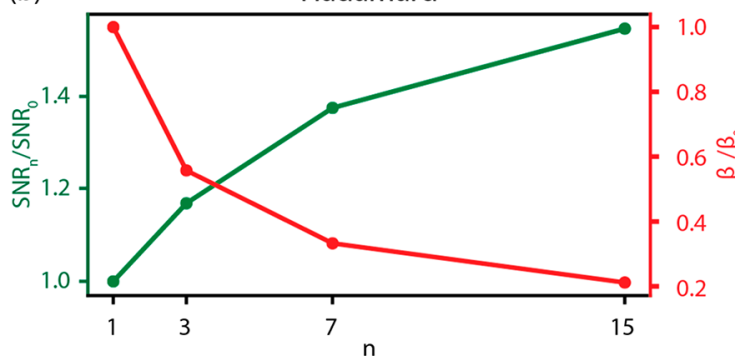

(c)
Black Light Sheet

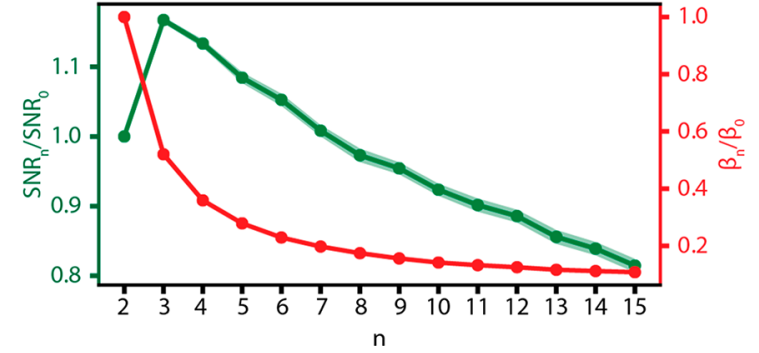

Figure 3. (a) Top: the same image of a single $1 \mu \mathrm{m}$ fluorescent bead taken with three different encoding methods (sequential, black light-sheet, and Hadamard scan). Bottom: Intensity profile, averaged over the columns, of the corresponding images. (b) Quantitative analysis of the normalized SNR gain (green) and background suppression (red) obtained with the Hadamard encoding. (c) Quantitative analysis of the normalized SNR gain (green) and background suppression (red) obtained with the black light-sheet encoding. The error bar is shown as a shaded area, when bigger than the line width. The images have been acquired with a $40 \times / 0.8 \mathrm{NA}$ objective lens, whose DoF has been extended from 1 to $60 \mu \mathrm{m}$ with the TAG lens.

linearity of its control system granted the desired uniformity without the need for any additional correction. Other lightsheet parallelization techniques have already been proposed, ${ }^{32,33}$ but acousto-optic light sculpting enables the fastest switch between an illumination pattern and the following one, ${ }^{34}$ which is key for preserving the speed of LSFM. In particular, in our current implementation, we can switch between two patterns within $5 \mathrm{~ms}$. This value does not depend on the number of produced beams; thus, in principle, it is possible to reach any amount of parallelization without sacrificing speed. Importantly, this value could be significantly decreased by implementing an optimized control system, and thus, it does not represent an inherent limitation of the technique. Indeed, the generation of the light-sheets in principle is limited only by the access time of the deflectors. $^{34,35}$

MELM can be implemented with any DoF extending method. In our system, we conjugated the back focal plane of the detection objective lens with a varifocal lens. ${ }^{36}$ Specifically, we used a tunable acoustic gradient (TAG) lens that rapidly scans the object plane along the optical axis. ${ }^{37}$ If the camera exposure time is greater than the period $T=2 \pi / \omega$ of the axial scan (typically on the order of tens of microseconds), a dynamic and tunable EDoF is obtained. The resulting detection point spread function (PSF) of the microscope is

$$
h_{e}(x, y, z)=\frac{1}{T} \int_{0}^{T} h_{o}(x, y, z-\Delta z \cos (\omega t)) \mathrm{d} t
$$

where $h_{\mathrm{o}}$ is the native detection PSF of the system, and $\Delta z$ is half of the scanning range. Importantly, $\Delta z$ can be tailored by changing the driving parameters of the TAG lens, namely, the frequency and the voltage amplitude (see Figure S2). As shown in Figure $2 \mathrm{c}, \mathrm{d}$, the resulting time-averaged PSF is elongated along the optical axis, while preserving its width in the $x-y$ plane. However, the out-of-focus contributions of the axially scanned PSF produce a lateral broadening of the tails of the extended PSF, resulting in additional fluorescent background. In order to restore the diffraction-limited images, a deconvolution step is needed, as typically happens with all EDoF techniques. Since the lateral shape of extended PSF does not depend on the axial coordinate, it is possible to perform a simple 2D plane-by-plane deconvolution. This advantage significantly lowers the computational cost of the postprocessing step compared to other DoF extending methods. Figure $2 \mathrm{~d}$ also shows how the average intensity of the extended PSF is lower than the peak intensity of the native PSF. As discussed before, this is an inherent side effect of any DoF extending methods: the longer the EdoF, the lower the signal of each plane (see Supporting Information for a full derivation).

With the two modifications in place, image acquisition in MELM proceeds by shining the sample with a sequence of multiple light-sheets. The encoding method, the extension of the DoF, and the planes to be imaged are selected prior to launching the acquisition by selecting the driving frequencies of both AOD and TAG lens. Once the stack of encoded frames 

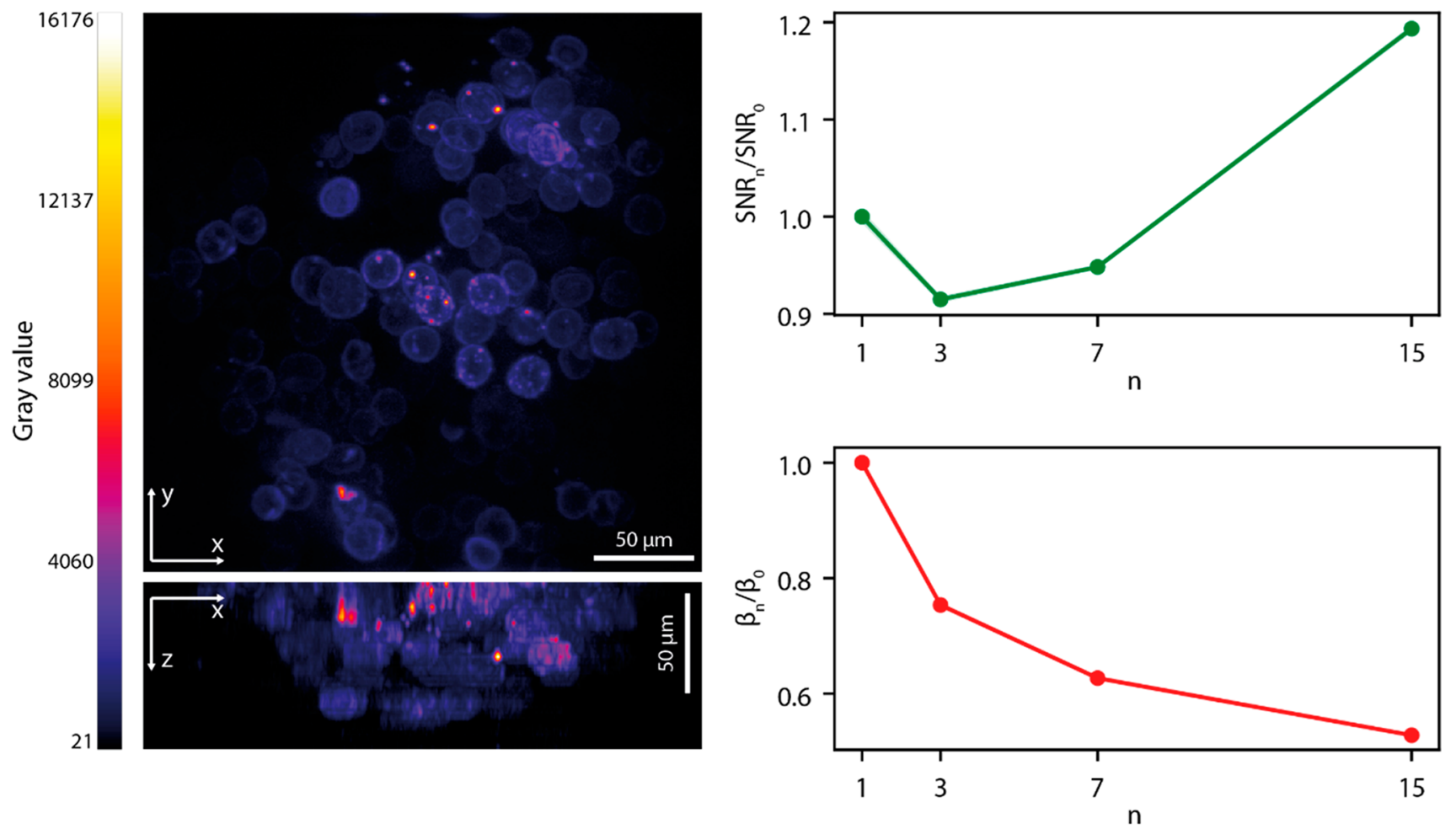

Figure 4. (a) Maximum intensity projection of a volumetric image of a spheroid acquired using the Hadamard encoding. The images have been deconvoluted with the Wiener filter algorithm, using a PSF simulated with eq 9. (c) SNR gain and background suppression versus the number of acquired frames. The images have been acquired with a 10×/0.3 NA objective lens, whose DoF has been extended from 7 to $215 \mu \mathrm{m}$ with the TAG lens. The 3D image is composed of two interdigitated stacks of 15 frames.

is collected, we reconstruct the images of the individual planes with a single-step decoding algorithm (see Materials and Methods section and Supporting Information for further details).

\section{FEASIBILITY OF MELM}

Initially, we validated our technique through numerical simulations. As shown in Figure S4, the results show a perfect agreement with theory and a clear benefit in terms of contrast and SNR. We next performed the imaging of fluorescent beads embedded in agar gel. Figure 3a shows the image of the same bead acquired with the sequential, black light-sheet, and Hadamard scan. For a fair comparison, all three cases were obtained using the same energy per light-sheet and the same camera exposure time. These parameters grant a comparable level of phototoxicity and the same acquisition speed. The exposure time has been set to $2 \mathrm{~ms}$, which added to the $5 \mathrm{~ms}$ required to switch between two encoding patterns, resulting in an acquisition rate of about 150 frames/s. As expected from such short exposure, the image acquired with the sequential scan is noisy and background-dominated. Instead, the black light-sheet scan shows a great suppression of the background, clearly noticeable in the intensity profile. However, there is no perceptible improvement in the noise level. With the Hadamard scan, the background level is not as low as with the previous encoding, but the noise suppression is significantly greater. Exact noise and background quantification has been performed using a Fourier-based algorithm, described in detail in the Materials and Methods section. The results are shown in Figure 3b,c, where we plot the SNR and contrast gain of both encoding methods as a function of the number of frames. The Hadamard scan provides an SNR gain monotoni- cally increasing with $n$, reaching an improvement of about $50 \%$ at $n=15$. Instead, the black light-sheet scan provides the maximum gain at $n=3$, which then decreases. Eventually, for high values of $n$, it loses its SNR advantage but maintains the background suppression. Both trends are in perfect agreement with the theoretical models discussed before.

A central benefit of MELM is its compatibility with any DoF extending method. To verify this aspect of the technique, we imaged a pollen grain with an EDoF generated by introducing spherical aberrations to our system. ${ }^{13}$ A widely used strategy given its simplicity, spherical aberrations produce an elongated PSF; however, it comes at the cost of losing uniformity. Indeed, the shape of the aberrated PSF strongly depends on the axial coordinate, thus requiring a more computationally expensive $3 \mathrm{D}$ deconvolution for image restoration. ${ }^{13,38}$ In this experiment, we increased spherical aberration by placing a slab of glass between a $40 \times / 0.8 \mathrm{NA}$ objective lens and the sample, resulting in an EDoF of about $30 \mu \mathrm{m}$. Note that, in contrast to varifocal lenses for EDoF, this technique is not tunable in realtime. Figure S6 shows images of a pollen grain obtained with the sequential, black light-sheet, and Hadamard scans for up to $n=7$ frames. The results are in line with those obtained with the TAG lens and in agreement with theory. Thus, the SNR gain is about $20 \%$ and the contrast gain about $300 \%$ using the Hadamard scan at $n=7$, and the black light-sheet offers the best background reduction of the three encoding strategies.

\section{IMAGING OF BIOLOGICAL SAMPLES}

As a proof of concept, we imaged a fluorescently labeled spheroid of human embryonic kidney cells. Given the superior SNR enhancement capabilities of Hadamard scan in the presence of shot noise, we selected this encoding sequence. 
Figure $4 \mathrm{a}$ shows the maximum intensity projections of a reconstructed volume of $278.5 \times 278.5 \times 83.7 \mu \mathrm{m}^{3}$, with a voxel size of $0.5 \times 0.5 \times 5.6 \mu \mathrm{m}^{3}(n=15)$. The camera exposure time was $100 \mathrm{~ms}$, and the volumetric imaging time was $1.5 \mathrm{~s}$. Under these conditions, and considering the dense labeling of spheroids, the photon noise is not negligible. Consequently, the enhancement in SNR is lower than that reported using fluorescent beads. A more in-depth analysis of the noise and background reduction is presented in Figure $4 \mathrm{~b}$. At the described experimental conditions, the SNR is still increasing with $n$, but for $n=3$ and $n=7$ it is lower than that of the sequential scan. However, for $n=15$, we reached an SNR gain of about $20 \%$ and a contrast gain of about $100 \%$. Significantly, such a gain comes with no cost in terms of 3D imaging speed.

Raising the number of frames $n$ could further increase the SNR gain, which is expected to approximately scale with the square root of the number of frames. However, practical limitations such as the resolution and the angular range of the deflector, or the power of the laser source, mitigate the feasibility of this strategy. In addition, some care must be taken when using our parallelization method. When the beams are generated, one should carefully avoid cross-talks. If beams are overlapping, a region of the sample will be illuminated by more than a light-sheet, failing the hypothesis of independent planes needed for the reconstruction. In this case, reconstruction is still possible, but it is likely to generate artifacts. On a positive note, MELM is an extremely efficient technique when it comes to illumination. Indeed, all available power of the light source can be used-the higher the power, the higher the number of planes that can be illuminated. Thus, we can maximize the photon budget and speed of EDoF-LSM and still preserve the very low photodamage of LSFM. In addition, even when the maximum number of illuminated planes is reached, it is still possible to acquire a volume of any arbitrary depth by performing a hybrid parallelized-sequential scan. It suffices to divide the imaged volume in $m$ stacks, each one composed of $n$ planes acquired in parallel. The resulting volume will be composed of $m \times n$ images with an improved SNR of about $\sqrt{n} / 2$.

\section{CONCLUSION}

The combination of EDoF detection with parallelized illumination enables overcoming the trade-off between speed and SNR in LSFM when low photon noise is present. Such a condition is typically encountered in fast volumetric imaging, where the exposure time is kept low. As our results demonstrate, illuminating the sample with sequences of multiple light-sheets produces images with enhanced SNR and contrast. Because no moving optics are being used, the intrinsic high speed of EDoF-LSFM is preserved. The encoding sequence can be selected to address the imaging needs of a particular sample or application. Furthermore, the increased SNR also enables smaller laser peak intensities, further decreasing the risk of photodamage. Thanks to the simplicity and versatility of MELM, it can be used with any DoF extending technique, making it suitable for any LSFM system. We expect MELM to become an invaluable tool to investigate dynamic biological processes at high spatiotemporal resolution in thick objects.

\section{MATERIALS AND METHODS}

Microscope Setup. The light source is a CW laser at 488 nm (Coherent Sapphire), beam-expanded with a $5 \times$ afocal telescope and then directed toward two AODs (IntraAction, ATD-7010CD2) at the Bragg angle. The deflectors are conjugated via a $4 \mathrm{f}$ system, thus generating a doubly diffracted beam which is then directed to the scan lens $(f=200 \mathrm{~mm})$, while the secondary beams are blocked by an iris. The beam is then aligned toward the tube lens $(f=200 \mathrm{~mm})$ and the excitation objective lens (Nikon $10 \times / 0.3 \mathrm{NA}$, water dipping), finally reaching the sample inside a chamber. The latter is a cube with transparent windows, filled with pure water, glued to the water dipping detection objective (Leica $10 \times / 0.3 \mathrm{NA}$ or $40 \times / 0.8 \mathrm{NA}$, both water dipping), mounted orthogonally to the excitation line. The back focal plane of the detection objective lens is conjugated through a $4 \mathrm{f}$ system to the TAG lens (TAG Optics Inc.). The TAG lens is typically driven at its first resonant frequency $(70 \mathrm{kHz})$, while the voltage amplitude depends on the desired extension of the DoF and the properties of the objective lens. After the TAG Lens, light is directed toward the tube lens $(f=200 \mathrm{~mm})$, which creates an image of the illuminated planes on the sensor of an sCMOS camera (ANDOR Neo 5.5). A notch filter (NF488-15, Thorlabs) rejects excitation light.

Acousto-Optic Light Sculpting. The first AOD, $\mathrm{AOD}_{y}$, (IntraAction, ATD-7010CD2) is used to deflect a Gaussian beam along the $y$-axis, thus generating a light-sheet on average. It is driven by a voltage-controlled oscillator (IntraAction, DE$704 \mathrm{M}$ ) at the central frequency of $70 \mathrm{MHz}$ with a $20 \mathrm{kHz}$ frequency modulation using a triangular wave. This waveform guarantees the optimal homogeneity of the light-sheet intensity along the $y$-axis. The second $\mathrm{AOD}, \mathrm{AOD}_{z}$, (IntraAction, $\mathrm{ATD}$ $7010 \mathrm{CD} 2)$ is driven by multiple frequencies in order to diffract the incident beam in multiple beamlets at different angles. The scan lens transforms the angles into unique axial positions, thus creating an array of parallel light-sheets. This AOD is controlled by a 14-bit arbitrary waveform generator (Signatec, PXDAC4800), whose output is amplified by a gain block (Mini-circuits, ZHL-1-2WX-S+). The camera acquisition and the generation of the driving signal for the $\mathrm{AOD}_{z}$ are synchronized through a digital $\mathrm{I} / \mathrm{O}$ device (National Instruments, USB-6501). The complete system is controlled by a custom software programmed with LabView. In its present form, the light-sculpting system can switch between two illumination sequences in $5 \mathrm{~ms}$.

Data Analysis. The images are decoded by multiplying the inverse of the encoding matrix with the array of acquired frames. For each experiment, an additional set of unencoded identical images is acquired in order to analyze them with the Fourier Ring Correlation (FRC) technique. ${ }^{39,40}$ The FRC algorithm provides an effective resolution value, which corresponds to a spatial frequency that we used as the cutoff for a high-pass digital filter. By applying this filter to the decoded $z$-stack, we obtained a map of the noise. We used the standard deviation of this map as a value of the noise content of the images. ${ }^{41}$ Furthermore, we applied a binarization algorithm to the decoded images in order to classify their content into signal or background (see Figure S3). Finally, when explicitly stated, we also applied a Wiener filter deconvolution algorithm to further improve the quality of the reconstructed $z$-stack. ${ }^{42}$ All the analysis has been 
performed via custom scripts programmed in Python. More details are available in the Supporting Information.

Sample Preparation. We prepared the bead samples using solutions of yellow-green beads (FluoSpheres, Invitrogen) with a diameter of $1 \mu \mathrm{m}$, diluted in $2 \%$ agarose gel at a concentration of 1:500. We prepared spheroids samples using human embryonic kidney cells (HEK 293), stably transfected with the plasmid encoding for EGFP- $\Delta 50$ lamin-A (Addgene plasmid \#17653). Starting from a dish of confluent cells, we diluted them to form a suspension of 10,000 cells $/ \mathrm{mL}$, and we seeded them into a 96-well microplate (Corning Spheroid Microplate). After 3 days of growth, we fixed the spheroids with $4 \%$ paraformaldehyde for $30 \mathrm{~min}$ at room temperature and cleared with Rapiclear 1.47 for $10 \mathrm{~min}$ (Sun Jin Lab Co.). Once prepared, we embedded the individual spheroids in a $2 \%$ agarose gel cylinder for imaging.

\section{ASSOCIATED CONTENT}

\section{SI Supporting Information}

The Supporting Information is available free of charge at https://pubs.acs.org/doi/10.1021/acsphotonics.1c01401.

Extended theoretical and experimental analysis (PDF) Movie 1: acousto-optic generation of multiple parallel light-sheets (MP4)

Movie 2: 3D reconstruction of a spheroid (MP4)

\section{AUTHOR INFORMATION}

\section{Corresponding Authors}

Alberto Diaspro - CHT, Istituto Italiano di Tecnologia, 16152 Genova, Italy; Department of Physics, University of Genoa, 16146 Genova, Italy; Email: alberto.diaspro@iit.it

Martí Duocastella - CHT, Istituto Italiano di Tecnologia, 16152 Genova, Italy; Department of Applied Physics, Universitat de Barcelona, 08028 Barcelona, Spain; - orcid.org/0000-0003-4687-8233;

Email: marti.duocastella@ub.edu

\section{Authors}

Alessandro Zunino - CHT, Istituto Italiano di Tecnologia, 16152 Genova, Italy; Department of Physics, University of Genoa, 16146 Genova, Italy

Francesco Garzella - CHT, Istituto Italiano di Tecnologia, 16152 Genova, Italy; SMFI Department, University of Parma, 43124 Parma, Italy

Alberta Trianni - CHT, Istituto Italiano di Tecnologia, 16152 Genova, Italy; Department of Physics, University of Genoa, 16146 Genova, Italy

Peter Saggau - CHT, Istituto Italiano di Tecnologia, 16152 Genova, Italy; Baylor College of Medicine, Houston, Texas 77030, United States

Paolo Bianchini - CHT, Istituto Italiano di Tecnologia, 16152 Genova, Italy; (1) orcid.org/0000-0001-6457-751X

Complete contact information is available at:

https://pubs.acs.org/10.1021/acsphotonics.1c01401

\section{Notes}

The authors declare no competing financial interest.

\section{ACKNOWLEDGMENTS}

The authors thank Luca Pesce for the HEK cell line and Giuseppe Vicidomini, Giorgio Tortarolo, and Eli Slenders for their assistance and for useful discussions. Dr. Duocastella is a
Serra Húnter fellow. This research was funded by Compagnia di San Paolo, grant agreement number 2019.0963 (Codice ROL 34704).

\section{REFERENCES}

(1) Stelzer, E. H. K. Light-Sheet Fluorescence Microscopy for Quantitative Biology. Nat. Methods 2015, 12 (1), 23-26.

(2) Cella Zanacchi, F.; Lavagnino, Z.; Perrone Donnorso, M.; Del Bue, A.; Furia, L.; Faretta, M.; Diaspro, A. Live-Cell 3D SuperResolution Imaging in Thick Biological Samples. Nat. Methods 2011, 8 (12), 1047-1050.

(3) Girkin, J. M.; Carvalho, M. T. The Light-Sheet Microscopy Revolution. J. Opt. (Bristol, U. K.) 2018, 20 (5), 053002.

(4) Fahrbach, F. O.; Rohrbach, A. Propagation Stability of SelfReconstructing Bessel Beams Enables Contrast-Enhanced Imaging in Thick Media. Nat. Commun. 2012, 3, 1 DOI: 10.1038/ncomms1646.

(5) Vettenburg, T.; Dalgarno, H. I. C.; Nylk, J.; Coll-Lladó, C.; Ferrier, D. E. K.; Čižmár, T.; Gunn-Moore, F. J.; Dholakia, K. LightSheet Microscopy Using an Airy Beam. Nat. Methods 2014, 11 (5), 541-544.

(6) Jia, H.; Yu, X.; Yang, Y.; Zhou, X.; Yan, S.; Liu, C.; Lei, M.; Yao, B. Axial Resolution Enhancement of Light-Sheet Microscopy by Double Scanning of Bessel Beam and Its Complementary Beam. J. Biophotonics 2019, 12 (1), 1-10.

(7) Huisken, J.; Swoger, J.; Del Bene, F.; Wittbrodt, J.; Stelzer, E. H. K. Optical Sectioning Deep inside Live Embryos by Selective Plane Illumination Microscopy. Science (Washington, DC, U. S.) 2004, 305 (5686), 1007-1009.

(8) Voleti, V.; Patel, K. B.; Li, W.; Perez Campos, C.; Bharadwaj, S.; Yu, H.; Ford, C.; Casper, M. J.; Yan, R. W.; Liang, W.; Wen, C.; Kimura, K. D.; Targoff, K. L.; Hillman, E. M. C. Real-Time Volumetric Microscopy of in Vivo Dynamics and Large-Scale Samples with SCAPE 2.0. Nat. Methods 2019, 16 (10), 1054-1062.

(9) Chakraborty, T.; Chen, B.; Daetwyler, S.; Chang, B. J.; Vanderpoorten, O.; Sapoznik, E.; Kaminski, C. F.; Knowles, T. P. J.; Dean, K. M.; Fiolka, R. Converting Lateral Scanning into Axial Focusing to Speed up Three-Dimensional Microscopy. Light: Sci. Appl. 2020, 9 (1), 1 DOI: 10.1038/s41377-020-00401-9.

(10) Truong, T. V.; Holland, D. B.; Madaan, S.; Andreev, A.; Keomanee-Dizon, K.; Troll, J. V.; Koo, D. E. S.; McFall-Ngai, M. J.; Fraser, S. E. High-Contrast, Synchronous Volumetric Imaging with Selective Volume Illumination Microscopy. Commun. Biol. 2020, 3 (1), 1-8.

(11) Wagner, N.; Norlin, N.; Gierten, J.; de Medeiros, G.; Balázs, B.; Wittbrodt, J.; Hufnagel, L.; Prevedel, R. Instantaneous Isotropic Volumetric Imaging of Fast Biological Processes. Nat. Methods 2019, 16 (6), 497-500.

(12) Woringer, M.; Darzacq, X.; Zimmer, C.; Mir, M. Faster and Less Phototoxic 3D Fluorescence Microscopy Using a Versatile Compressed Sensing Scheme. Opt. Express 2017, 25 (12), 13668.

(13) Tomer, R.; Lovett-Barron, M.; Kauvar, I.; Andalman, A.; Burns, V. M.; Sankaran, S.; Grosenick, L.; Broxton, M.; Yang, S.; Deisseroth, K. SPED Light Sheet Microscopy: Fast Mapping of Biological System Structure and Function. Cell 2015, 163 (7), 1796-1806.

(14) Olarte, O. E.; Andilla, J.; Artigas, D.; Loza-Alvarez, P. Decoupled Illumination Detection in Light Sheet Microscopy for Fast Volumetric Imaging. Optica 2015, 2 (8), 702.

(15) Quirin, S.; Peterka, D. S.; Yuste, R. Instantaneous ThreeDimensional Sensing Using Spatial Light Modulator Illumination with Extended Depth of Field Imaging. Opt. Express 2013, 21 (13), 16007.

(16) Fahrbach, F. O.; Voigt, F. F.; Schmid, B.; Helmchen, F.; Huisken, J. Rapid 3D Light-Sheet Microscopy with a Tunable Lens. Opt. Express 2013, 21 (18), 21010.

(17) Duocastella, M.; Sancataldo, G.; Saggau, P.; Ramoino, P.; Bianchini, P.; Diaspro, A. Fast Inertia-Free Volumetric Light-Sheet Microscope. ACS Photonics 2017, 4 (7), 1797-1804. 
(18) Kang, S. Y.; Duocastella, M.; Arnold, C. B. Variable Optical Elements for Fast Focus Control. Nat. Photonics 2020, 14 (9), 533542.

(19) Chaze, W.; Caballina, O.; Castanet, G.; Lemoine, F. The Saturation of the Fluorescence and Its Consequences for LaserInduced Fluorescence Thermometry in Liquid Flows. Exp. Fluids 2016, 57 (4), 1-18.

(20) Tinevez, J. Y.; Dragavon, J.; Baba-Aissa, L.; Roux, P.; Perret, E.; Canivet, A.; Galy, V.; Shorte, S. A Quantitative Method for Measuring Phototoxicity of a Live Cell Imaging Microscope, 1st ed.; Elsevier Inc., 2012; Vol. 506. DOI: 10.1016/B978-0-12-391856-7.00039-1.

(21) Icha, J.; Weber, M.; Waters, J. C.; Norden, C. Phototoxicity in Live Fluorescence Microscopy, and How to Avoid It. BioEssays 2017, 39 (8), $1-15$.

(22) Judkewitz, B.; Yang, C. Axial Standing-Wave Illumination Frequency-Domain Imaging (SWIF). Opt. Express 2014, 22 (9), 11001.

(23) Ren, Y.; Wu, J.; Lai, Q. T. K.; Lai, H. M.; Siu, D. M. D.; Wu, W.; Wong, K. K. Y.; Tsia, K. K. Parallelized Volumetric Fluorescence Microscopy with a Reconfigurable Coded Incoherent Light-Sheet Array. Light: Sci. Appl. 2020, 9 (8), 1 DOI: 10.1038/s41377-0200245-8.

(24) Diaspro, A. Taking Three-Dimensional Two-Photon Excitation Microscopy Further: Encoding the Light for Decoding the Brain. Microsc. Res. Tech. 2013, 76 (10), 985-987.

(25) Ducros, M.; Houssen, Y. G.; Bradley, J.; De Sars, V.; Charpak, S. Encoded Multisite Two-Photon Microscopy. Proc. Natl. Acad. Sci. U. S. A. 2013, 110 (32), 13138-13143.

(26) Wuttig, A. Optimal Transformations for Optical Multiplex Measurements in the Presence of Photon Noise. Appl. Opt. 2005, 44, 2710.

(27) Nitzsche, G.; Riesenberg, R. Noise, Fluctuation and HADAMARD-Transform-Spectrometry. Proc. SPIE 2003, 5111, 273-282.

(28) Harwit, M.; Sloane, N. J. A.; Sloane, I.; James, N. Hadamard Transform Optics 1979, 96.

(29) Gavryusev, V.; Sancataldo, G.; Ricci, P.; Montalbano, A.; Fornetto, C.; Turrini, L.; Laurino, A.; Pesce, L.; de Vito, G.; Tiso, N.; Vanzi, F.; Silvestri, L.; Pavone, F. S. Dual-Beam Confocal Light-Sheet Microscopy via Flexible Acousto-Optic Deflector. J. Biomed. Opt. 2019, 24 (10), 1.

(30) Keller, P. J.; Schmidt, A. D.; Wittbrodt, J.; Stelzer, E. H. K. Reconstruction of Zebrafish Early Embryonic Development by Scanned Light Sheet Microscopy. Science (Washington, DC, U. S.) 2008, 322 (5904), 1065-1069.

(31) Trypogeorgos, D.; Harte, T.; Bonnin, A.; Foot, C. Precise Shaping of Laser Light by an Acousto-Optic Deflector. Opt. Express 2013, 21 (21), 24837.

(32) Mohan, K.; Purnapatra, S. B.; Mondal, P. P. Three Dimensional Fluorescence Imaging Using Multiple Light-Sheet Microscopy. PLoS One 2014, 9 (6), 1-8.

(33) Dean, K. M.; Roudot, P.; Welf, E. S.; Pohlkamp, T.; Garrelts, G.; Herz, J.; Fiolka, R. Imaging Subcellular Dynamics with Fast and Light-Efficient Volumetrically Parallelized Microscopy. Optica 2017, 4 (2), 263

(34) Duocastella, M.; Surdo, S.; Zunino, A.; Diaspro, A.; Saggau, P. Acousto-Optic Systems for Advanced Microscopy. J. Phys. Photonics 2021, 3, 012004.

(35) Zunino, A.; Surdo, S.; Duocastella, M. Dynamic Multifocus Laser Writing with Acousto-Optofluidics. Adv. Mater. Technol. 2019, 4 (12), 1-7.

(36) Martinez-Corral, M.; Hsieh, P. Y.; Doblas, A.; Sanchez-Ortiga, E.; Saavedra, G.; Huang, Y. P. Fast Axial-Scanning Widefield Microscopy with Constant Magnification and Resolution. J. Disp. Technol. 2015, 11 (11), 913-920.

(37) McLeod, E.; Arnold, C. B. Mechanics and Refractive Power Optimization of Tunable Acoustic Gradient Lenses. J. Appl. Phys. 2007, 102 (3), 033104.
(38) Wallace, W.; Schaefer, L. H.; Swedlow, J. R. A Workingperson's Guide to Deconvolution in Light Microscopy. BioTechniques 2001, 31 (5), 1076-1097.

(39) Koho, S.; Tortarolo, G.; Castello, M.; Deguchi, T.; Diaspro, A.; Vicidomini, G. Fourier Ring Correlation Simplifies Image Restoration in Fluorescence Microscopy. Nat. Commun. 2019, 10 (1), 1 DOI: $10.1038 / \mathrm{s} 41467-019-11024-z$.

(40) Tortarolo, G.; Castello, M.; Diaspro, A.; Koho, S.; Vicidomini, G. Evaluating Image Resolution in Stimulated Emission Depletion Microscopy. Optica 2018, 5 (1), 32.

(41) Liu, S.; Mlodzianoski, M. J.; Hu, Z.; Ren, Y.; McElmurry, K.; Suter, D. M.; Huang, F. SCMOS Noise-Correction Algorithm for Microscopy Images. Nat. Methods 2017, 14 (8), 760-761.

(42) Gonzalez, R. C.; Woods, R. E. Digital Image Processing, 2nd ed.; Prentice Hall, 2002. 